\title{
Method for Optimizing the Kinematic Scheme of Stretch-Forming Parts from Sheet Blanks
}

\author{
Konstantin Bormotin ${ }^{1, *}$, Anton Krivenok ${ }^{2}$, Maria Pogartseva ${ }^{1}$, and Min Ko Hlaing ${ }^{1}$ \\ ${ }^{1}$ Komsomolsk-na-Amure State University, 681013, Komsomolsk-on-Amur, Russia \\ ${ }^{2}$ Komsomolsk- na -Amure Aviation Plant named after Yu.A. Gagarina, 681018, Komsomolsk-on- \\ Amur, Russia
}

\begin{abstract}
The modeling of the stretch-forming process a sheet on a punch is considered. A numerical method has been developed for solving the optimal control problems of the stretch-forming process sheet blanks. The values of the residual displacements are considered as an optimization criterion. The algorithms implemented in $\mathrm{CAE}$ allow calculating the optimal parameters for the operation of the $\mathrm{CNC}$ stretch-forming press. The simulation results are compared with full-scale experiments on the shaping of sheet parts on a stretch-forming press T-600.
\end{abstract}

\section{Introduction}

One of the ways to obtain details of aircraft structures is the stretch-forming process. These are details such as skins and frame elements, which are obtained by bending with tension from sheet and profile blanks on numerically controlled stretch presses. The accuracy of manufacturing details by the stretch forming method is greatly influenced by such technological conditions of the shaping process as the geometry of the punch and the kinematics of the stretch forming press control bodies.

At the present time, the stretch forming punch is manufactured according to electronic models of details without taking into account the material springback. In this case, the elastic recovery of the material (springback) can have a significant effect on the accuracy of the detail manufacture. In practice, in order to minimize deviations of the formed workpiece from the given geometry, at the last stage of wrapping, calibrating tension with a high level of elongation is applied, up to rupture of the workpiece. This solution leads to a decrease in the plastic resource of the workpiece material and does not always provide the required shaping accuracy.

Existing software tools and methods for calculating control programs do not always provide accurate manufacturing of details, which increases their production cycle due to manual revision and the search for optimal modes of shaping and adjusting control programs. To reduce the production preparation time, it is necessary, when creating control programs, to take into account the stress-strain state of the workpiece material during its deformation according to the loading scheme.

\footnotetext{
* Corresponding author: cvmi@knastu.ru
} 
Various methods of stretch forming on presses are proposed: taking into account the displacements of the workpiece relative to the punch [1,2], taking into account a certain sequence of the stretch forming kinematic schemes with stages alternating with loading and unloading $[3,4]$.

CAE-systems makes it possible to simulate the stretch forming processes that implement various deformation schemes and select the best ones [5-7], but for a complete analysis of possible solutions, it is necessary to develop a method for finding the optimal shaping scheme.

The calculation of the tooling, which provides the necessary anticipatory sheet shape, can be performed by the iterative method for solving inverse problems [8, 9]. As an optimization method, the method of dynamic programming is considered, implemented in finite element algorithms for solving [10].

The results of modeling and optimization are compared with the data of full-scale experiments carried out on a T-600 press for shaping the skin of an aircraft detail.

\section{Formulation and method for solving problem}

Let $V \subset R^{3}$ be a bounded domain with boundary $S$. Area for setting edge displacements sheet blank $-S_{b}$. Denote by $u=\left(u_{1}, u_{2}, u_{3}\right), \tilde{u}=\left(\tilde{u}_{1}, \tilde{u}_{2}, \tilde{u}_{3}\right)-$ is the vectors of current and residual displacements of deformable body, $t$ - is the parameter of deformation.

It is assumed that some form of the punch is known. In this case, the problem of kinematic shaping will include the problem of deformation in plasticity and elastic unloading. The problems of mechanics are formulated by variational principles with functionals:

$$
J(\dot{u})=W_{c}+a(\dot{u}, \dot{u}) \text { at }\left.\dot{u}\right|_{S_{b}}=\dot{u}^{*}, J(\dot{\tilde{u}})=W_{c}+a(\dot{\tilde{u}}, \dot{\tilde{u}})
$$

where $W_{c}-$ is the contact potential [11, 12]; potential form are given by $a(\dot{u}, \dot{v})=\int_{V} \frac{\partial E\left(\dot{u}_{i, j}\right)}{\partial \dot{u}_{i, j}} \dot{v}_{i, j} d V, \quad E\left(\dot{u}_{i, j}\right)=\frac{1}{2} c_{i j k l} \dot{\varepsilon}_{i j} \dot{\varepsilon}_{k l}-c_{i j k l} \dot{\varepsilon}_{i j} \dot{\varepsilon}_{k l}^{p}+\frac{1}{2} \sigma_{i j} \dot{u}_{k, i} \dot{u}_{k, j}$, $a(\dot{\tilde{u}}, \dot{\tilde{v}})=\int_{V}\left(\frac{\partial \tilde{E}\left(\dot{\tilde{u}}_{i, j}\right)}{\partial \dot{\tilde{u}}_{i, j}}\right) \dot{\tilde{v}}_{i, j} d V, \quad \tilde{E}\left(\dot{\tilde{u}}_{i, j}\right)=\frac{1}{2} c_{i j k l} \dot{\tilde{\varepsilon}}_{i j} \dot{\tilde{\varepsilon}}_{k l}-c_{i j k l} \dot{\tilde{\varepsilon}}_{i j} \dot{\varepsilon}_{k l}^{p}+\frac{1}{2} \rho_{i j} \dot{\tilde{u}}_{k, i} \dot{\tilde{u}}_{k, j}, \quad c_{i j k l}$ - are the components of the elastic constant tensor; $\dot{\varepsilon}_{k l}^{p}$ - are the plastic strain rates [12], $\dot{\varepsilon}_{i j}, \dot{\tilde{\varepsilon}}_{i j}$ - are the velocity components of the current and residual strains of Green-Lagrange, $\sigma_{i j}, \rho_{i j}$ are the current and residual components of the second stress tensor of Piola-Kirchhoff, $u_{i, j}=\frac{\partial u_{i}}{\partial x_{j}}, i, j, k, l=1,2,3$.

The problem of optimal deformation is to find the movement trajectory of the clamps in the stretch forming process on the punch, which ensured the minimum deviations of the sheet after unloading from the surface of the punch. This problem includes the equations of the deformable solid mechanics, obtained from the stationarity conditions (1), and the optimization functional

$$
J=\int_{S} \sum_{\alpha}\left(\tilde{u}_{\alpha}(T)-\tilde{u}_{\alpha}^{*}\right)^{2} d S \rightarrow \inf
$$


where $\alpha$ - is the component of residual displacements, which determines the panel deflection, $\tilde{u}_{\alpha}{ }^{*}$ - is the given components of the sheet displacement to the punch, ensuring the adherence of the sheet to the punch surface.

Taking into account the discretization of variation (1), finite element equations are formed to solve the contact problem $[11,12,13]$

$$
{ }^{t+\Delta t} \mathbf{K} \Delta \mathbf{U}={ }^{t+\Delta t} \mathbf{R},{ }^{t+\Delta t} \tilde{\mathbf{K}} \Delta \hat{\mathbf{U}}={ }^{t+\Delta t} \hat{\mathbf{R}},
$$

where ${ }^{t+\Delta t} \mathbf{K},{ }^{t+\Delta t} \tilde{\mathbf{K}}$ - are the tangent stiffness matrices (in matrices are already included the additional elements which are formed from contact restrictions), ${ }^{t+\Delta t} \mathbf{R},{ }^{t+\Delta t} \hat{\mathbf{R}}$ - are the vectors of internal and external forces. The top indexes $t+\Delta t$ of quantity indicate time for which it is calculated. Residual nodal displacements are determined by the formula $\tilde{\mathbf{U}}=\mathbf{U}+\hat{\mathbf{U}}$.

For an approximate solution of the optimal control problem, the interval $[0, T]$ is divided into $N$ parts: $0=t_{0}<t_{1}<t_{2}<\ldots<t_{N}=T$. Using time-discrete equations of the step-by-step integration procedure (3), provided $\Delta t \leq \Delta t^{\prime}=t_{k+1}-t_{k}$, stresses, strains and displacements are calculated. For the case under consideration, criterion (2) will be evaluated by a discrete analog of the functional in the form

$$
J=\sum_{S} \sum_{\alpha}\left(\left(\sum_{k=0}^{N-1} \sum_{t=t_{k}}^{t_{k+1}} \Delta \tilde{\mathbf{U}}_{\alpha}(t)\right)-\tilde{\mathbf{U}}_{\alpha}^{*}\right) \rightarrow \inf ,
$$

where $\Delta \tilde{\mathbf{U}}_{\alpha}(t)-\alpha$-components of the nodes residual displacements increment caused by the increment of current displacements. Due to the springback of the sheet, the value in brackets (4) will be positive.

Equations (3) and optimization criteria (4) form discrete optimal control problem. In such a formulation, the Bellman function is constructed and the problem is solved by the dynamic programming method [14].

\section{Numerical results of solving the optimization problem and experimental data}

The solution of the modeling and optimization problem of the sheet stretch forming process is carried out by the finite element method in MSC.Marc (Fig.1.). Due to symmetry, only the right side of the sheet is considered. A workpiece with a thickness of $3 \mathrm{~mm}$ and a length of $1000 \mathrm{~mm}$ is specified by a set of 4-angle elements for 2D analysis (plane strain conditions). The contact interaction of bodies at the loading stage a workpiece with a punch is determined with a friction coefficient equal to 0.1 . The workpiece has the properties of material 1163T: Young's modulus $E=7073 \mathrm{kgf} / \mathrm{mm}^{2}$, Poisson's ratio $v=0.34$, yield stress $\sigma_{T}=30 \mathrm{kgf} / \mathrm{mm}^{2}$, hardening is given by the power law model.

Vector-function $\mathbf{U}(t)$ of points panel displacements on the boundary $S_{b}$ of clamp is specified radius and corner in the form $\mathbf{U}_{x}(t)=\mathbf{U}_{x}\left(f_{1}(t) \cdot r^{*}, f_{2}(t) \cdot \varphi^{*}\right)$, $\mathbf{U}_{y}(t)=\mathbf{U}_{y}\left(f_{1}(t) \cdot r^{*}, f_{2}(t) \cdot \varphi^{*}\right) \quad\left(\mathbf{U}(t)=\left(\mathbf{U}_{x}(t), \mathbf{U}_{y}(t), 0\right)\right), \quad$ where $\quad \mathbf{U}_{x}^{*}=\mathbf{U}_{x}\left(r^{*}, \varphi^{*}\right)$, 
$\mathbf{U}_{y}{ }^{*}=\mathbf{U}_{y}\left(r^{*}, \varphi^{*}\right)$ - final displacement values, $f_{1}(t) \in[0,1], f_{2}(t) \in[0,1]$. It is necessary to find the optimal functional dependence of the displacement components $\mathbf{U}_{y}=f\left(\mathbf{U}_{x}\right)$.

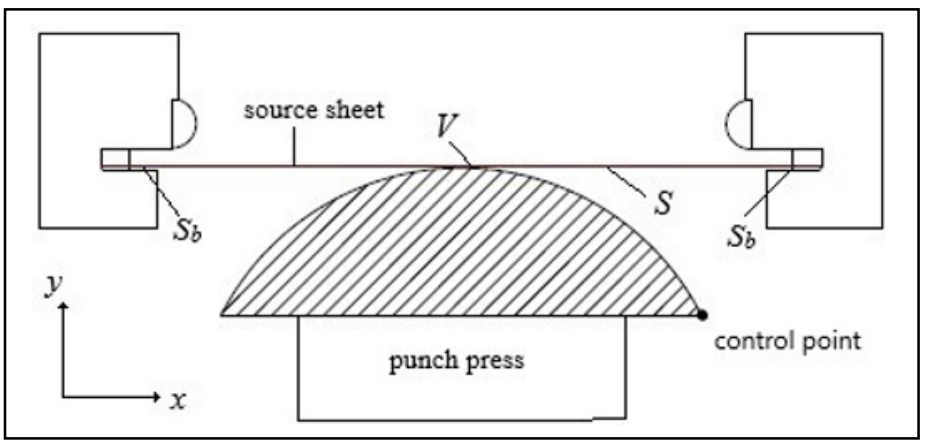

Fig. 1. Stretch-forming press model.

To use Bellman recurrence relations, a grid is constructed in space $(r, \varphi)$. The step along the radius $r$ is given and equal $\Delta_{r}$, along the angle $\varphi-\Delta_{\varphi}$. When denoting grid nodes by $P_{m}(n)$, the index $n$ will mean the number of the hyperplane $\Sigma_{n}$ for a given value of $r$, and the index $m$ - the number of the node in the hyperplane $\Sigma_{n}$.

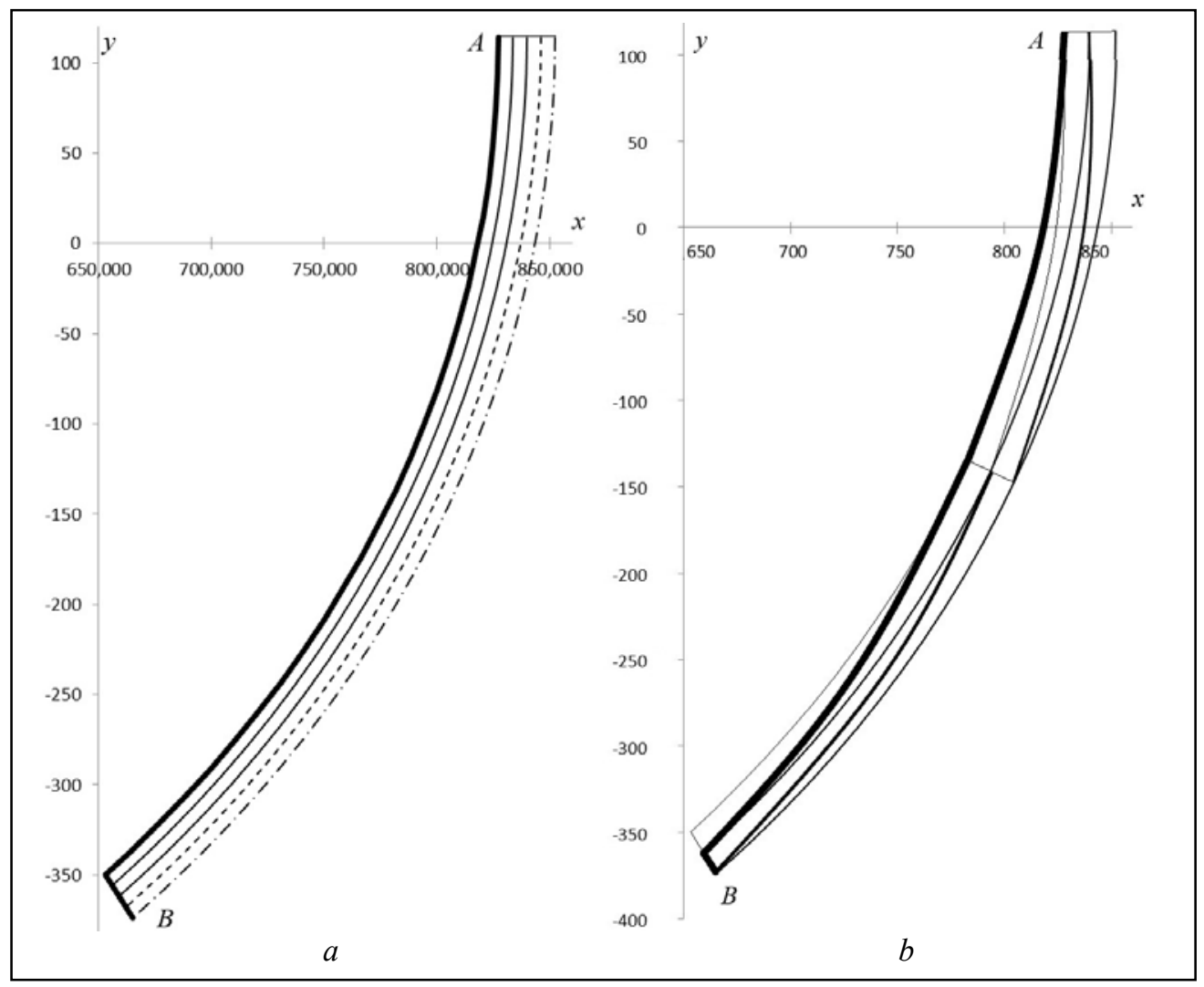

Fig. 2. Possible options and optimal trajectory of the press clamping movement when the workpiece is deformed. 
As a result of such operations, one can obtain a graph in which nodes $P_{m}(n)$ play the role of vertices, and instead of the original problem, the problem of finding on this graph the shortest path connecting the hyperplanes $\Sigma_{0}$ and $\Sigma_{N}$ will be considered.

Denoting by $l_{m}(n)$ the broken line of the shortest length connecting the node $P_{m}(n)$ with the hyperplane $\Sigma_{0}$, one can arrive at the recurrence relation [14]:

$$
l_{s}(n+1)=\min _{m}\left\{l_{m}(n)+l_{m s}(n)\right\} .
$$

The minimum is taken over those numbers $m$ for which the nodes lie in the feasible region $G_{n}$ and belong to the hyperplane $\Sigma_{n}$. The implementation of the dynamic programming method for this problem is similar to [10].

Fig. 2 shows variants of deformation trajectories from point $A$ to $B$ for the right edge of the sheet when stretch-formed with a mesh at $N=4, M=1$ and $N=2, M=2$ (the optimal path is highlighted in bold). The displacements are indicated in $\mathrm{mm}$. Trajectory curves are approximated by elliptical arcs. In the case of the grid $N=2, M=2$, as can be seen, the optimal trajectory deviates from the ellipse curve (Fig. 2b). In criterion (4), instead of the workpiece surface, the workpiece point with maximum displacements (control point) is considered.

Full-scale experiments were carried out on a stretch-forming press T-600 using equipment for shaping the skin of an aircraft detail (Fig. 1). The workpieces are strips from a sheet of $1163 \mathrm{~T}$ material $200 \mathrm{~mm}$ wide, $2000 \mathrm{~mm}$ long and $3 \mathrm{~mm}$ thick. The experimental results were carried out for three loading trajectories (bold, dashed and dash-dotted curves in Fig. 2a) and are presented in Fig. 3, and in comparison with modeling - in Fig. 4. These trajectories correspond to three loading schemes: bending - stretching (B-S), stretching bending - stretching (S-B-S) and stretching - bending (S-B).

Fig. 4 shows the deviations of the control point after unloading the sheet, depending on measurements and samples in comparison with the results of modeling for three loading schemes. Experimental studies carried out to assess the reliability of the results of mathematical modeling showed a practical coincidence for various schemes of stretchforming sheet details on a T-600 press.

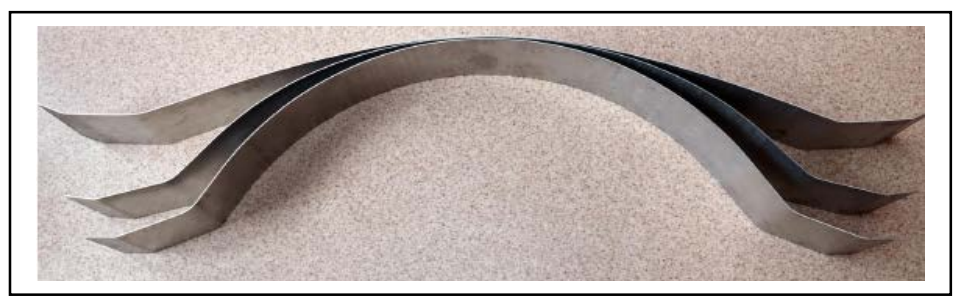

Fig. 3. Samples of workpiece stretch-formed on a T-600 press according to different loading schemes

\section{Conclusions}

In the considered optimization method, the original problem is reduced to a sequence of auxiliary simpler minimization problems. This method allows us to consider various loading paths and at the same time reduces the amount of calculations compared to a simple enumeration of all possible deformation paths, since non-optimal paths are excluded during the calculation. According to the results of modeling and full-scale experiments, the optimal kinematic shaping scheme is bending followed by stretching.

The developed numerical method, implemented in the CAE-system, makes it possible to take into account the complex geometry of the detail, material properties and makes it 
possible, even at the stage of production preparation, to optimize the shaping parameters to reduce the cycle of the technological process.

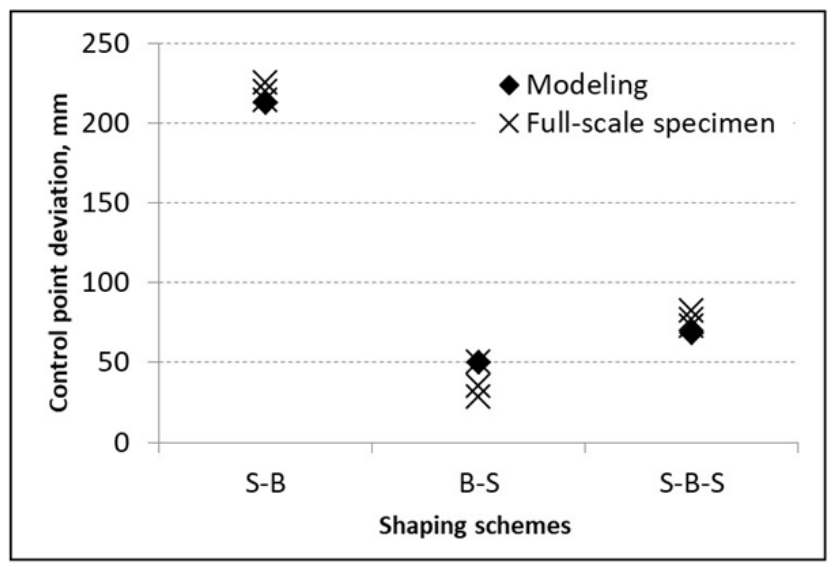

Fig. 4. Deviations of full-scale specimen at the control point from adherence to the punch in comparison with the results of numerical modeling.

This research was financially supported by the Russian Science Foundation (grant № 21-11-00165).

\section{References}

1. M. Molod, Bulletin of Voronezh State Technical University, 12-2, 7, 62-64(2011)

2. S.V. Belykh, A.A. Krivenok, V.V. Mironenko, V.A. Mishagin, Vestnik Irkutskogo gosudarstvennogo tehnicheskogo universiteta, 12(83), 36-40 (2013)

3. V.A. Miheev, F.V. Grechnikov, S.G. Dementyev, V.P. Samohvalov, D.V. Savin, S.V. Surudin, Izvestia of Samara Scientific Center of the Russian Academy of Sciences, 16, 6,172-179 (2014)

4. V.A. Miheev, Y.S. Klochkov, A.A. Kuzina, A.F. Grechnikova, D.V. Savin, Vestnik Samarskogo gosudarstvennogo aerokosmicheskogo universiteta, 5 (36), 239-245 (2012)

5. A. Kolesnikov, V. Mironenko, A. Cheslavskaya, A. Shmakov, Vestnik Irkutskogo gosudarstvennogo tehnicheskogo universiteta, 12 (83), 73-77 (2013)

6. V.V. Mironenko, A.A. Cheslavskaya, S.V. Belykh, Scholarly notes of Komsomolskna-Amure state technical university, II-1(18), 13-18 (2014)

7. Jingwen Peng, Weidong Li, Jinquan Han, Min Wan, Bao Meng, Int J Adv Manuf Technol, 86, 3571-3582 (2016)

8. K. Bormotin, Win Aung, MATEC Web of Conferences, 224, 04014 (2018)

9. K.S. Bormotin, Vychislitel'nye Metody i Programmirovanie, 18,359-370 (2017)

10. K.S. Bormotin, Win Aung, IOP Conference Series: Materials Science and Engineering, 709(3), 033102 (2020)

11. P. Wriggers, Computational Contact Mechanics (Springer-Verlag Berlin Heidelberg, 2006)

12. S.N. Korobeinikov, Nonlinear Deformation of Solids (Novosibirsk, Izd. Sib. Otd. Ross. Akad. Nauk, 2000)

13. Marc 2016, Vol A: Theory and User Information, MSC.Software Corporation http://www.mscsoftware.com/product/marc.

14. F.P. Vasil'ev, Optimization Methods (Moscow, Faktorial Press, 2002). 